OPEN ACCESS

Edited by:

Massimiliano Veroux,

University of Catania, Italy

Reviewed by:

Ibrahim Mohamed Elzayat,

Aswan University, Egypt

Zhihong Wang,

China Medical University, China

Panayiotis Economides,

European University Cyprus, Cyprus

*Correspondence:

Hui Sun

s_h@jlu.edu.cn

Haixia Guan

hxguan@vip.126.com

${ }^{\dagger}$ These authors have contributed equally to this work and share

last authorship

Specialty section:

This article was submitted to

Surgical Oncology,

a section of the journal

Frontiers in Oncology

Received: 30 August 2021 Accepted: 03 December 2021 Published: 22 December 2021

Citation:

Li C, Dionigi G, Liang N, Guan $H$ and Sun $H$ (2021)

The Relationship Between Body

Mass Index and Different Regional Patterns of Lymph Node Involvement

in Papillary Thyroid Cancers.

Front. Oncol. 11:767245.

doi: 10.3389/fonc.2021.767245

\section{The Relationship Between Body Mass Index and Different Regional Patterns of Lymph Node Involvement in Papillary Thyroid Cancers}

\author{
Changlin Li $^{1}$, Gianlorenzo Dionigi ${ }^{2,3}$, Nan Liang $^{1}$, Haixia Guan ${ }^{4,5 * t}$ and Hui Sun ${ }^{1 * t}$ \\ ${ }^{1}$ Division of Thyroid Surgery, China-Japan Union Hospital of Jilin University, Jilin Provincial Key Laboratory of Surgical \\ Translational Medicine, Jilin Provincial Engineering Laboratory of Thyroid Disease Prevention and Control, Changchun \\ City, China, ${ }^{2}$ Division of Surgery, Istituto Auxologico Italiano IRCCS, Milan, Italy, ${ }^{3}$ Department of Medical Biotechnology and \\ Translational Medicine, University of Milan, Milan, Italy, ${ }^{4}$ Department of Endocrinology, Guangdong Provincial People's \\ Hospital, Guangdong Academy of Medical Sciences, Guangzhou, China, ${ }^{5}$ The Second School of Clinical Medicine, Southern \\ Medical University, Guangzhou, China
}

Introduction: Lymph node (LN) metastasis is the first site of metastasis of papillary thyroid cancer (PTC). LN status influences clinical management and the prognosis of patients. We explored the relationship between patient obesity and regional patterns of $L N$ involvement in PTC.

Materials and Methods: This study retrospectively analyzed the data from 12,772 PTC patients. The rate of $L N$ metastasis, number of $L N$ metastasis, maximum diameter of positive $L N$, number of dissected $L N$, and $L N$ ratios $(L N R)$ were compared between normal-weight and obese patients. Statistical methods have been adjusted for the confounders in hypothesis testing.

Results: Overweight and obesity were independent risk factor for metastatic $\mathrm{LNs}\left(\mathrm{OR}_{1}=\right.$ $\left.1.125,95 \% \mathrm{Cl} 1.042-1.214, P_{1}=0.003 ; \mathrm{OR}_{2}=1.554,95 \% \mathrm{Cl} 1.339-1.802, P_{2}<0.001\right)$. Obesity was an independent risk factor for the number of metastatic CLNs (OR=1.159, 95\% Cl 0.975-1.377, $P=0.045$ ), however not for number of metastatic lateral LNs $(P=0.907)$. Furthermore, obesity was not an independent risk factor for number of CLNs when dissected more than five $(P=0.653)$, still an independent risk factors for number of metastatic lateral LNs when more than six (OR=1.185, 95\% Cl 1.010-1.391, $P=0.037)$. As for $L N R$, obesity was an independent risk factor for the central LNR when more than $0.12\left(\mathrm{OR}_{\text {adjusted } 1}=1.099,95 \% \mathrm{Cl} 1.011-1.194, P_{1}=0.027 ; \mathrm{OR}_{\text {adjusted } 2}=\right.$ $\left.1.177,95 \% \mathrm{Cl} 1.003-1.381, P_{2}=0.045\right)$, for the lateral LNR more than $0.05\left(P_{2}=0.283\right)$.

Conclusions: Obesity was associated with poor prognoses with PTC respecting LNs. Surgeons should be extreme caution when performing central neck dissection in obese patients.

Keywords: body mass index, lymph node, papillary thyroid cancer, metastasis, regional patterns 


\section{INTRODUCTION}

The prevalence of obesity is approximately $40 \%$ worldwide, affecting more than 2 billion adults (1). Obesity has been identified as an independent risk factor for many cancers. Some studies have reported that nearly $40 \%$ of cancers may be attributed to obesity (2), and there is strong evidence to suggest that obesity is related to cancers of the esophagus, liver, pancreas, gallbladder, ovary, thyroid, kidneys, and plasma cells (3). Although obesity has been linked to an increased risk of diabetes and coronary artery disease, the impact of obesity on the incidence rates, risk factors, morbidity, and mortality of thyroid cancer requires further exploration (4). As thyroid cancer is the most common type of endocrine tumor, understanding how BMI impacts this disease has vital public health implications (5). Obesity also affects the diagnostic assessment of patients negatively. Deglise et al. found that obese women were less likely to have undergone ultrasound $(\mathrm{OR}=0.5)$ or MRI $(\mathrm{OR}=0.3)$ and were at an increased risk of prolonged hospital stays $(\mathrm{OR}=4.7)$ in the clinic (6).

A significant association has been established between elevated BMIs and increased papillary thyroid carcinoma (PTC) incidence rates (7). In a previous study, obesity was identified as a risk factor for thyroid cancer, specifically when tumor sizes were larger than $1 \mathrm{~cm}$ with multifocality and extrathyroidal extensions (8-10). However, the connection between obesity and lymph node (LN) metastasis of thyroid cancer has not been thoroughly investigated. One of the most important prognostic factors in PTC is LN status (11). LN metastasis is the first station of extra-gland metastasis of thyroid cancer (11). Hence, LN metastasis is an excellent predictor of the prognosis of patients with thyroid cancer. LN status can influence important clinical decisions, such as therapeutic options (11). The American Thyroid Association (ATA) guidelines use the number of metastatic LNs and the maximum diameter of positive LNs as important indicators for predicting the risk of recurrence (11). Compared to patients with less than five metastatic LNs, the recurrence rates are much higher for patients with more than five metastatic LNs (19\% vs. $8 \%)(12,13)$. In terms of maximum positive LN diameter, recurrence rates are significantly higher in patients with the diameter of LNs higher than $3 \mathrm{~cm}$ (27\% vs. $5 \%$ ) (13).

In this study, we focused on the relationship between obesity and the patterns of LN involvement in PTC, with an emphasis on the number of metastatic LNs, the maximum diameter of positive LNs, the lymph node ratio (LNR), the number of dissected LNs, and the LN skip metastasis.

\section{MATERIALS AND METHODS}

\section{Study Design}

This study was a retrospective descriptive analysis of patients with operable PTC. The patients in this study were diagnosed with PTC at our Institute between June 2008 and December 2017. Patient data was gathered and stored in the Institutional database of the Division of Thyroid Surgery, China-Japan Union Hospital of Jilin University. The Institutional data collected have important impact locally on the epidemiological surveillance, evaluation of thyroidal disease, impact of the treatment on diseases progression and improvement on research quality.

All the patients eligible for this analysis received central neck node dissection. Patients were aged $\geq 18$ years, pathologicallyconfirmed to have PTC, and PTC patients routinely underwent prophylactic central neck dissection (CND). The exclusion criteria was: non-PTC patients, other thyroid cancer subtypes, different types of cancer, family history of thyroid cancer; history of cervical radiation exposure in adolescence or childhood, incomplete data, no lymph node dissection, and patients requiring reoperations (Figure 1). Patients who do not fall into the criteria of this study will be excluded from the effectiveness data set, but all safety observation records will be kept in a safe center.

\section{Treatments}

Oncological treatment, which ranged from surgery to radioactive iodine (RAI), was standardized for all patients and in accordance with the multidisciplinary tumor board consensus. The histological subtype was assessed according to the WHO classification. According to the Chinese guidelines for diagnosis and treatment of differentiated thyroid, all patients with thyroid cancer routinely underwent prophylactic CND (14). For those LNs suggested to be malignant by ultrasonography, fine needle aspiration cytology (FNAC) was used to confirm the diagnosis. Patients with cervical lymph node metastasis were

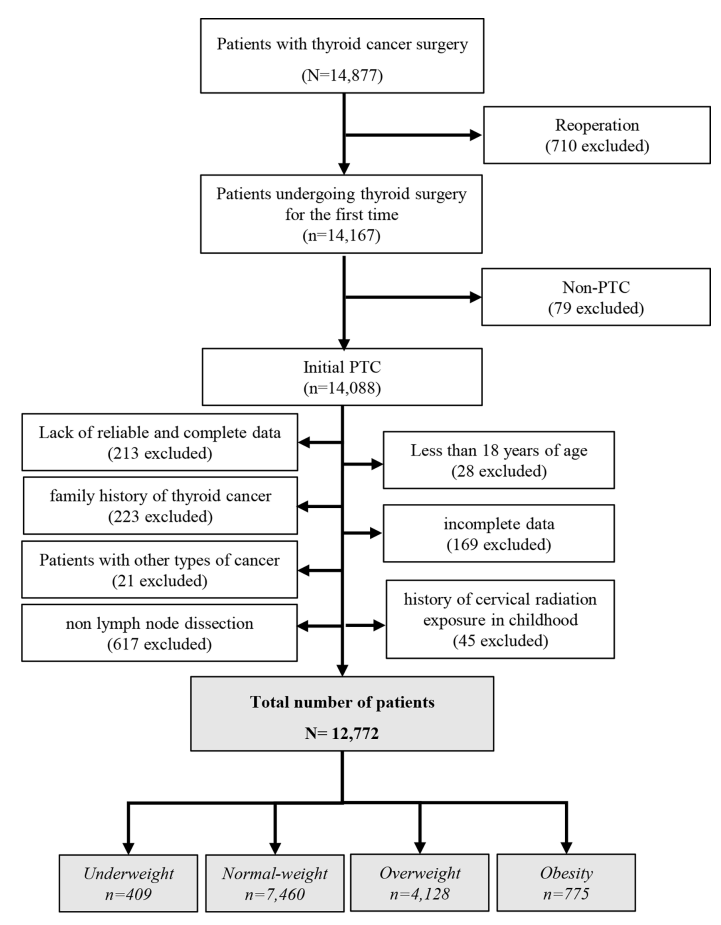

FIGURE 1 | Flow chart of study inclusion and exclusion criteria. 
confirmed by preoperative FNAC or intraoperative frozen pathological examinations. These patients underwent therapeutic cervical lymph node dissection. Prophylactic cervical lymph node dissection was not recommended. The upper bound of the range of CND is the lower hyoid bone, the lower bound is the superior sternum fossa, the outside is the common carotid artery, and the inside is the inside of the trachea. The lateral LN dissection minimum ranged from the IIa, III, IV, and $\mathrm{Vb}$ area, while other areas were treated according to the results of the FNAC.

\section{Definitions}

Body-mass index. According to the WHO-BMI standard, underweight is $<18.5 \mathrm{~kg} / \mathrm{m}^{2}$, normal weight is $18.5-24.9 \mathrm{~kg} / \mathrm{m}^{2}$, overweight is $25-29.9 \mathrm{~kg} / \mathrm{m}^{2}$, and obese is $\geq 30 \mathrm{~kg} / \mathrm{m}^{2}$ (15). Height and weight measurements used to calculate the BMI were retrieved from electronic registration databases. BMI was calculated as weight divided by height squared. Upon the first admission, the demographics and clinical data, including height and weight, were recorded.

Pattern of metastatic LNs. The pattern of metastatic LNs included the rate of metastatic LNs, the number of metastatic LNs, the number of dissected LNs, the lymph node ratio (LNR), the maximum diameter of positive LNs, and the rate of LN skip metastasis.

Maximum diameter of positive LNs. The maximal tumor diameter of the largest metastatic LN using the concept of micro-metastases in breast cancer (16).

Lymph node ratio (LNR). LNR was defined as the number of nodes involved by the tumor divided by the total number of resected lymph nodes during the surgical treatment.

LN skip metastasis. Defined as a lateral lymph node metastasis without central lymph node involvement.

\section{Pathological Examination}

Specimens removed during the operation were submitted for histological analysis to determine the presence and size of metastatic LNs. For this study, an experienced pathologist reviewed the pathological slides and measured the properties of each LN.

\section{Outcomes and Covariates}

We used BMI as an index and further correlated it with the presence and pattern of LN metastases. Patients were identified as LN positive if they had a pathologic LN status of $\mathrm{pN1a}$ or pN1b. Based on prior studies, we included important predictors of LN status in multivariable models (17). For example, information regarding the following thyroid tumor characteristics was obtained from medical records: age, weight, height, tumor size ( $\mathrm{mm})$, multifocality, extrathyroidal extension, rate of metastatic LNs (\%), number of metastatic LNs, number of dissected LN, LNR (\%), maximum diameter of positive LNs ( $\mathrm{mm})$, and LN skip metastasis (\%).

\section{Sample Size Collection}

Retrospective analysis of sample size (i.e., margin of error and level of confidence calculation) was carried out through
"Raosoft ${ }^{\circledR}$ sample size calculator" (http://www.raosoft.com/ samplesize.html), on the basis of the Chinese epidemiologic data recently published (3). This reporting an overall number of 90.000 new cases of thyroid cancer for the year 2015, assuming that $90 \%$ of these tumors are represented by DTC with a $20 \%$ rate of relapse after thyroidectomy and iodine therapy.

\section{Statistical Analysis}

All data were collected using Microsoft Excel (Microsoft Corporation, Redmond, WA, USA). Continuous variables were expressed as mean (standard deviation), and categorical variables were expressed as a percentage (frequency). Continuous variables were analyzed by the $t$-test or one-way analysis of variance (ANOVA). Categorical variables were examined using the $\chi^{2}$-test or Fischer's exact test. Binary logistic regression calculates the dependent variable OR and 95\% confidence interval (CI). The characteristics of pathological invasion were regarded as dependent variables, WHO-BMI group was used as a covariate, and age, sex, FT3, TPO-Ab, tumor diameter $>1 \mathrm{~cm}$, multifocality, High $\mathrm{T}$ stage and extrathyroidal extension were used as adjustment variables. TSH, FT3, FT4, TPO-Ab and Tg$\mathrm{Ab}$ do not conform to the normal distribution, so logarithmic transformation is performed. $P<0.05$ was considered statistically significant. Statistical analysis was performed using SPSS 22.0 software (IBM, Chicago, IL, USA).

\section{RESULTS}

\section{Baseline Characteristics of Patients}

Our database included 14,877 patients with PTC, of which 12,772 patients were included in the final analysis (Figure 1). The male-to-female ratio is $1: 3.7$. The average age is $42.73 \pm 9.41$ years. The average BMI is $24.27 \pm 3.49 \mathrm{~kg} / \mathrm{m}^{2}$. Obesity accounted for $6.1 \%$ of patients. In total, 166,802 LNs were dissected, of which 24,670 LNs were metastases $(14.8 \%)$. The rate of metastatic LNs was $44.9 \%(5437 / 12,772)$. The mean number of $\mathrm{LN}$ metastases was $1.93 \pm 3.81$. The average number of $\mathrm{LN}$ dissected was $13.06 \pm 12.31$, and the LNR was $0.14 \pm 0.23 \%$ as shown in Table 1.

\section{Influencing Factors of Metastatic LNs}

Single factor logistic regression was used to analyze the factors influencing metastatic LNs. We identified other confounding factors related to the presence of metastatic LNs in addition to overweight and obesity. As shown in Table 2, age, FT3, $\mathrm{TPO}-\mathrm{Ab}$, tumor diameter more than $1 \mathrm{~cm}$, multifocality, High $\mathrm{T}$ stage and extrathyroidal extension are confounding factors for metastatic LNs.

\section{Impact of BMI on the Rate of Metastatic LNs}

As shown in Table 3, the rate of metastatic LNs in overweight and obesity patients with PTCs was significantly higher than that of normal-weight patients ( 45.9 vs. 52.6 vs. $42.0 \%, P<0.001$ ). The rates of central metastatic LNs and lateral neck metastatic LNs were significantly higher in overweight and obesity patients than 
TABLE 1 | Basic demographics and clinical data of patients included in this study.

\section{Characteristic}

Total $(\mathrm{N}=12,772)$ Mean (SD) or \%(n)

\section{Sex}

Female (\%)

Male (\%)

Mean age (years)

Mean BMl $\left(\mathrm{kg} / \mathrm{m}^{2}\right)$

$\mathrm{BMl}$ group (WHO-BMI)

Underweight (\%)

Normal-weight (\%)

Overweight (\%)

Obesity (\%)

Thyroid Function

$\mathrm{TSH}(\mathrm{mlU} / \mathrm{L})$

FT3 (pmol/L)

FT4 (pmol/L)

$\mathrm{Tg}-\mathrm{Ab}(\mathrm{IUmL})$

TPO-Ab (IUmL)

Mean tumor size (mm)

Multifocality

Extrathyroidal extension

Rate of total metastatic LN (\%)

Number of total LN+

Number of total dissected $L N$

Total LNR

$$
\begin{gathered}
78.9 \%(10,073) \\
21.1 \%(2,699) \\
42.73(9.41) \\
24.27(3.49) \\
\\
3.2 \%(409) \\
58.4 \%(7,460) \\
32.3 \%(4,128) \\
6.1 \%(775) \\
\\
3.20(3.43) \\
4.53(1.06) \\
15.80(5.79) \\
\\
0.83(0.62) \\
40.9 \%(5,230) \\
26.9 \%(3,441) \\
44.9 \%(5,737) \\
1.93(3.81) \\
13.06(12.31) \\
0.14(0.23)
\end{gathered}
$$

BMI, body mass index; TSH, thyroid stimulating hormone; FT3, free triiodothyronine; FT4, free thyroxine; $L N$, lymph node; $L N R$, lymph node ratio.

normal-weight patients $(41.5 \%$ vs. 47.6 vs. $38.0 \%, P<0.001 ; 18.8$ vs. 22.2 vs. $16.9 \%, P<0.001)$. The rates of lateral neck metastatic LNs on both the left and right sides were higher than normalweight patients ( 10.2 vs. 11.1 vs. $8.6 \%, P=0.008 ; 10.5$ vs. 13.7 vs. 9.8\%, $P<0.001)$.

Binary logistic regression analyzed the relationship between BMI and the risk of metastatic LNs. It was found that overweight and obesity were independent risk factor for metastatic LNs $\left(\mathrm{OR}_{1}=1.125,95 \% \mathrm{CI} 1.042-1.214, P_{1}=0.003 ; \mathrm{OR}_{2}=1.554,95 \%\right.$ CI 1.339-1.802, $P_{2}<0.001$ ) (Table 4). According to the central and lateral neck lymph nodes, the adjusted OR value of metastatic LNs was calculated respectively. It was found that overweight and obesity were independent risk factor for metastatic CLNs (OR adjusted $1=1.129,95 \%$ CI 1.038-1.227, $\left.P_{1}=0.005 ; \mathrm{OR}_{\text {adjusted } 2}=1.174,95 \% \mathrm{CI} 1.001-1.378, P_{2}=0.049\right)$. After adjusting for confounding factors, overweight and obesity were not independent risk factors for metastatic lateral LNs $\left(P_{1}=\right.$ 0.621, $P_{2}=0.657$ ) (Table 4).

\section{Impact of BMI on the Number of Metastatic LNs}

As shown in Table 3, the total number of LN metastases was significantly higher in obese patients than normal-weight patients $(2.41$ vs. $1.79, P<0.001)$. Among the obese patients, the number of LN metastases in the central and lateral neck regions were higher than the normal-weight patients (1.59 vs. $1.17, P<0.001 ; 0.83$ vs. $0.62, P=0.006)$. In addition, the number of lateral neck LN metastases was higher than that of the normalweight patients on the left side ( 0.40 vs. $0.27, P=0.018)$.

It was found that overweight and obesity were independent risk factor for metastatic LNs more than three $(\mathrm{OR}$ adjusted $1=$ $1.108,95 \%$ CI 1.011-1.214, $P_{1}=0.029 ; \mathrm{OR}_{\text {adjusted } 2}=1.6225 \% \mathrm{CI}$ 1.377-1.910 $P_{2}<0.001$ ) (Table 5). It was found that overweight and obesity were independent risk factor for number of metastatic CLNs more than two $\left(\mathrm{OR}_{1}=1.106,95 \%\right.$ CI 1.006$1.215, P_{1}=0.038 ; \mathrm{OR}_{2}=1.159,95 \%$ CI $\left.0.975-1.377, P_{2}=0.045\right)$. After adjusting for confounding factors, overweight and obesity were not independent risk factors for number of metastatic lateral LNs more than two $\left(P_{1}=0.866, P_{2}=0.907\right)$ (Table 5).

\section{Impact of BMI on the Number of Dissected LNs}

The total number of dissected $L N$ in the obese patients with PTC was significantly higher than the normal-weight patients (14.37 vs. $12.10, P<0.001)$. The obese patients have a higher total

TABLE 2 | Regression analysis of influencing factors of metastatic LNs.

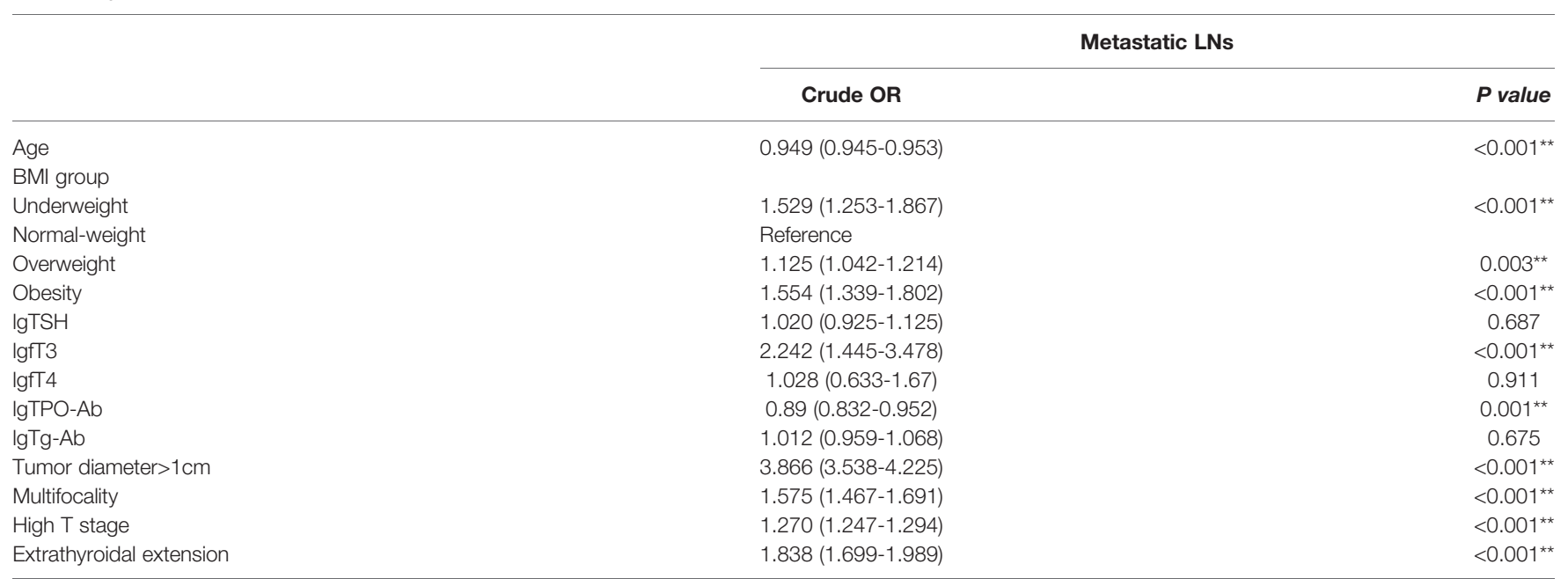

BMI, body mass index; TSH, thyroid stimulating hormone; FT3, free triiodothyronine; FT4, free thyroxine; OR, oods ratio; LN, lymph node. ${ }^{*} P<0.05,{ }^{* *} P<0.01$. 
TABLE 3 | Relationship between obesity and different regional patterns of lymph node involvement.

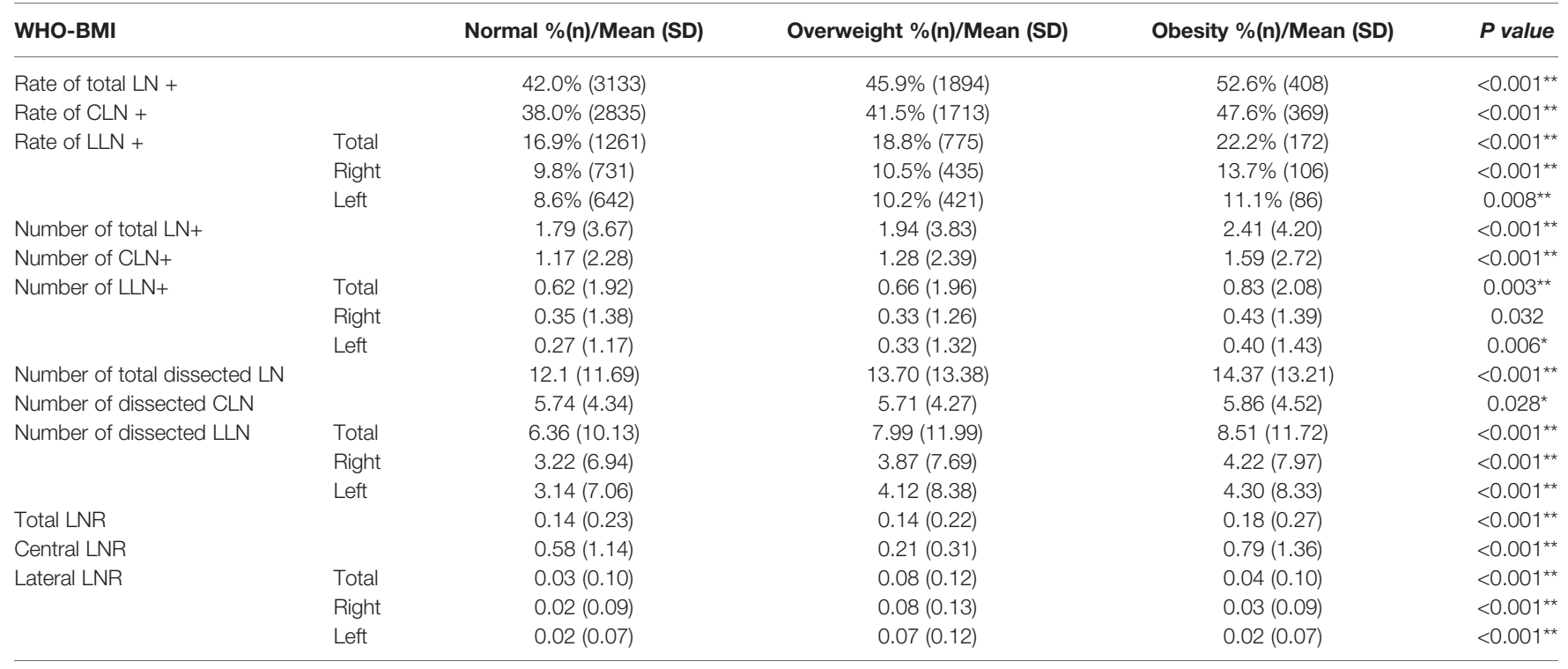

$L N+$, lymph node metastasis; CLN +, central lymph node metastasis; $L L N+$, lateral lymph node metastasis.

${ }^{*} P<0.05,{ }^{* *} P<0.01$.

number of lateral neck dissected $L N(8.51$ vs. 6.36, $P<0.001)$. This difference exists on the left and right sides of the neck (Table 4). However, there was no difference in the number of CNDs $(P=0.466)$.

Binary logistic regression analyzed the relationship between BMI and number of dissected LNs. Overweight and obesity were independent risk factor for the number of dissected LNs more than twelve $\left(\mathrm{OR}_{1}=1.156,95 \% \mathrm{CI} 1.069-1.250, P_{1}<0.001 ; \mathrm{OR}_{2}=\right.$ $1.376,95 \%$ CI 1.184-1.59, $P_{2}<0.001$ ) (Table 6). It was found that obesity was not an independent risk factor for number of dissected CLNs more than five $(P=0.653)$. After adjusting for confounding factors, overweight and obesity were independent risk factors for number of dissected lateral LNs more than six $\left(\mathrm{OR}_{1}=1.122,95 \% \mathrm{CI} 1.032-1.219, P_{1}=0.007 ; \mathrm{OR}_{2}=1.185,95 \%\right.$ CI 1.010-1.391, $P_{2}=0.037$ ) (Table 6).

\section{Impact of BMI on the LNR}

The LNR in obese patients was significantly higher than normalweight patients $(0.18$ vs. $0.14, P<0.001)$ (Table 5). While the difference was statistically significant in the central LNs $(0.79$ vs. $0.58, P<0.001)$, but there was no statistically significant difference in the lateral neck LNs $(P=0.067)$ (Table 5).

It was found that overweight and obesity were independent risk factor for the LNR more than 0.07 (OR adjusted $1=1.086,95 \%$ CI 1.005-1.174, $P_{1}=0.036$; OR adjusted $2=1.537,95 \%$ CI 1.325 $1.783 P_{2}<0.001$ ) (Table 7). It was found that overweight and obesity were independent risk factor for the central LNR more than $0.12\left(\mathrm{OR}_{1}=1.099,95 \% \mathrm{CI} 1.011-1.194, P_{1}=0.027 ; \mathrm{OR}_{2}=\right.$ $1.177,95 \%$ CI $\left.1.003-1.381, P_{2}=0.045\right)$. But obesity was not an independent risk factor for the lateral LNR more than $0.05\left(P_{2}=\right.$ 0.283) (Table 7).

TABLE 4 | Binary logistic regression analyzed the relationship between BMl and the risk of metastatic LNs.

\begin{tabular}{|c|c|c|c|c|c|}
\hline WHO-BMI & & Underweight & Normal-weight & Overweight & Obesity \\
\hline \multirow[t]{4}{*}{ Risk of total metastatic LNs } & Crude OR & $1.529(1.253-1.867)$ & Reference & $1.125(1.042-1.214)$ & $1.554(1.339-1.802)$ \\
\hline & $P$ value & $<0.001^{\star \star}$ & & $0.003^{\star \star}$ & $<0.001^{\star \star}$ \\
\hline & Adjusted OR & $1.139(0.915-1.417)$ & Reference & 1.119 (1.03-1.216) & $1.198(1.02-1.408)$ \\
\hline & $P$ value & 0.244 & & $0.008^{\star \star}$ & $0.028^{\star}$ \\
\hline \multirow[t]{4}{*}{ Risk of metastatic CLNs } & Crude OR & 1.602 (1.312-1.955) & Reference & $1.120(1.036-1.21)$ & $1.511(1.303-1.752)$ \\
\hline & $P$ value & $<0.001^{\star \star}$ & & $0.004^{\star *}$ & $<0.001^{\star \star}$ \\
\hline & Adjusted OR & $1.164(0.937-1.447)$ & Reference & $1.129(1.038-1.227)$ & $1.174(1.001-1.378)$ \\
\hline & $P$ value & 0.171 & & $0.005^{\star \star}$ & $0.049^{\star}$ \\
\hline \multirow[t]{4}{*}{ Risk of metastatic LLNs } & Crude OR & $1.487(1.175-1.882)$ & Reference & 1.106 (1.002-1.22) & $1.436(1.203-1.715)$ \\
\hline & $P$ value & $0.001^{\star \star}$ & & $0.046^{\star}$ & $<0.001^{\star \star}$ \\
\hline & Adjusted OR & $1.17(0.9-1.521)$ & Reference & $1.028(0.923-1.144)$ & $1.045(0.86-1.271)$ \\
\hline & $P$ value & 0.242 & & 0.621 & 0.657 \\
\hline
\end{tabular}

BMI, body mass index; OR, odds ratio; Cl, confidence interval; $L N+$, lymph node metastasis; CLN, central lymph node; LLN, lateral lymph node. Age, IgFT3, IgTPO-Ab, tumor diameter $>1 \mathrm{~cm}$, multifocality, High $T$ stage and extrathyroidal extension as covariates to adjust OR value. ${ }^{*} P<0.05,{ }^{* *} P<0.01$. 
TABLE 5 | Binary logistic regression analyzed the relationship between BMl and the number of metastatic LNs.

\begin{tabular}{|c|c|c|c|c|c|}
\hline WHO-BMI & & Underweight & Normal-weight & Overweight & Obesity \\
\hline \multirow[t]{4}{*}{ Number ${ }^{1}$ of total metastatic LNs } & Crude OR & $1.859(1.502-2.301)$ & Reference & $1.108(1.011-1.214)$ & $1.622(1.377-1.910)$ \\
\hline & $P$ value & $<0.001^{\star \star}$ & & $0.029^{*}$ & $<0.001^{\star \star}$ \\
\hline & Adjusted OR & $1.294(1.017-1.648)$ & Reference & $1.091(0.987-1.207)$ & $1.201(1.002-1.439)$ \\
\hline & $P$ value & $0.036^{\star}$ & & 0.090 & $0.047^{\star}$ \\
\hline \multirow[t]{4}{*}{ Number $^{2}$ of metastatic CLNs } & Crude OR & $1.718(1.394-2.117)$ & Reference & $1.091(1.000-1.190)$ & $1.526(1.302-1.789)$ \\
\hline & $P$ value & $<0.001^{\star \star}$ & & 0.051 & $<0.001^{\star \star}$ \\
\hline & Adjusted OR & $1.148(0.91-1.448)$ & Reference & $1.106(1.006-1.215)$ & $1.159(0.975-1.377)$ \\
\hline & $P$ value & 0.243 & & $0.038^{\star}$ & $0.045^{\star}$ \\
\hline \multirow[t]{4}{*}{ Number ${ }^{3}$ of metastatic LLNs } & Crude OR & $1.665(1.287-2.154)$ & Reference & $1.076(0.96-1.206)$ & $1.447(1.184-1.769)$ \\
\hline & $P$ value & $<0.001^{\star \star}$ & & 0.208 & $<0.001^{\star \star}$ \\
\hline & Adjusted OR & $1.263(0.947-1.686)$ & Reference & 0.989 (0.874-1.12) & $1.013(0.813-1.262)$ \\
\hline & $P$ value & 0.112 & & 0.866 & 0.907 \\
\hline
\end{tabular}

BMI, body mass index; OR, odds ratio; Cl, confidence interval; LNs, lymph nodes; CLNs, central lymph nodes; LLNs, lateral lymph nodes.

Age, tumor diameter $>1 \mathrm{~cm}$, multifocality, High $T$ stage and extrathyroidal extension as covariates to adjust OR value.

${ }^{1}$ The cut-off point of number in total $L N+$ is three.

${ }^{2}$ The cut-off point of number in total CLN + is two.

${ }^{3}$ The cut-off point of number in total LLN + is two.

${ }^{*} P<0.05,{ }^{* *} P<0.01$.

\section{Impact of BMI on the Size of Positive LNs}

As shown in Figure 2A, the maximum diameter of positive LNs in obese patients was higher than normal-weight patients (2.00 vs. $1.60 \mathrm{~mm}, P=0.007)$. There was no significant difference in the rate of LN skip metastasis between the two groups (Figure 2B).

\section{DISCUSSION}

This study is a retrospective analysis of 12,772 patients with PTC, with a focus on the correlation between LN status and obesity. Obesity not only increased the rate of metastatic LNs, but also increased the number of metastatic LNs and the maximum diameter of positive LNs, which are two indicators of a poor prognosis. We analyzed two indicators that have been overlooked in previous studies, including the number of dissected $L N$ and the LNR. These results showed opposite regional differences between obesity, the number of dissected LN, and the LNR (Figure 3).

\section{Effect on the Rate of Metastatic LNs}

Previous studies on the relationship between obesity and metastatic LNs in thyroid cancer have been controversial (Table 8) (18-28). Yu et al. found a positive correlation between $\mathrm{BMI}$ and neck LN metastasis $(\mathrm{OR}=1.58, P=0.02)$ (24). Our previous research also yielded consistent results with those of Yu et al. $(\mathrm{OR}=1.493)(8,23,24)$. One of the possible mechanisms is that serum leptin levels are higher in obese patients with PTC. In vitro studies have indicated that leptin promotes invasion and migration of thyroid cancer cell lines (29, 30). However, Kim, Gasior, Grani, Tresallet, and others have found that obesity is not associated with neck metastatic LNs in patients with thyroid cancer $(19,20,26,28)$. Simultaneously, Paes et al. found that obesity is negatively correlated with metastatic LNs (18). This finding may be due to differences in ethnicity. In the current study, we found that the rate of metastatic LNs in obese patients with PTC was significantly higher than normal-weight patients $(52.6 \%$ vs. $42.0, P<0.001)$.

TABLE 6 | Binary logistic regression analyzed the relationship between BMl and the number of $L$ Ns dissections.

\begin{tabular}{|c|c|c|c|c|c|}
\hline WHO-BMI & & Underweight & Normal-weight & Overweight & Obesity \\
\hline \multirow[t]{4}{*}{ Number of total LNs dissections ${ }^{1}$} & Crude OR & $1.317(1.076-1.611)$ & Reference & $1.156(1.069-1.250)$ & $1.376(1.184-1.598)$ \\
\hline & $P$ value & $0.007^{\star \star}$ & & $<0.001^{* *}$ & $<0.001^{\star *}$ \\
\hline & Adjusted OR & $0.992(0.808-1.218)$ & Reference & $1.128(1.038-1.226)$ & $1.173(1-1.377)$ \\
\hline & $P$ value & 0.942 & & $0.004^{\star}$ & $0.047^{\star}$ \\
\hline \multirow[t]{4}{*}{ Number of CLNs dissections ${ }^{2}$} & Crude OR & $1.16(0.95-1.416)$ & Reference & $0.875(0.81-0.945)$ & $1.127(0.971-1.307)$ \\
\hline & $P$ value & 0.145 & & $0.001^{\star \star}$ & 0.115 \\
\hline & Adjusted OR & $0.964(0.782-1.189)$ & Reference & $0.895(0.826-0.97)$ & $1.036(0.888-1.208)$ \\
\hline & $P$ value & 0.735 & & $0.007^{\star \star}$ & 0.653 \\
\hline \multirow[t]{4}{*}{ Number of LLNs dissections ${ }^{3}$} & Crude OR & $1.26(1.029-1.542)$ & Reference & $1.168(1.08-1.264)$ & $1.384(1.191-1.607)$ \\
\hline & $P$ value & $0.025^{\star}$ & & $<0.001^{\star \star}$ & $<0.001^{\star \star}$ \\
\hline & Adjusted OR & $1.14(0.918-1.414)$ & Reference & $1.122(1.032-1.219)$ & $1.185(1.010-1.391)$ \\
\hline & $P$ value & 0.235 & & $0.007^{\star \star}$ & $0.037^{\star}$ \\
\hline
\end{tabular}

BMI, body mass index; OR, odds ratio; LN, lymph node; CLN, central lymph node; LLN, lateral lymph node.

Age, IgFT3, IgTPO-Ab, tumor diameter $>1 \mathrm{~cm}$, multifocality, High T stage and extrathyroidal extension as covariates to adjust OR value.

${ }^{1}$ The cut-off point of number in total LNs dissections is twelve.

${ }^{2}$ The cut-off point of number in total CLNs dissections is five.

${ }^{3}$ The cut-off point of number in total LLNs dissections is six.

${ }^{*} P<0.05,{ }^{* *} P<0.01$. 
TABLE 7 | Binary logistic regression analyzed the relationship between BMl and the risk of LNR.

\begin{tabular}{|c|c|c|c|c|c|}
\hline WHO-BMI & & Underweight & Normal-weight & Overweight & Obesity \\
\hline & $P$ value & $<0.001^{\star *}$ & & $0.036^{\star}$ & $<0.001^{\star \star}$ \\
\hline \multirow[t]{3}{*}{ Central LNR ${ }^{2}$} & Crude OR & $1.526(1.25-1.862)$ & Reference & $1.095(1.014-1.184)$ & $1.511(1.303-1.752)$ \\
\hline & $P$ value & $<0.001^{* \star}$ & & $0.021^{\star}$ & $<0.001^{\star \star}$ \\
\hline & Adjusted OR & $1.106(0.89-1.374)$ & Reference & 1.099 (1.011-1.194) & $1.177(1.003-1.381)$ \\
\hline & $P$ value & $<0.001^{\star \star}$ & & $<0.001^{\star \star}$ & $0.002^{\star \star}$ \\
\hline & Adjusted OR & $19.425(15.183-24.854)$ & Reference & 18.239 (16.501-20.16) & $1.113(0.915-1.354)$ \\
\hline & $P$ value & $<0.001^{\star \star}$ & & $<0.001^{\star \star}$ & 0.283 \\
\hline
\end{tabular}

OR, odds ratio; LNR, lymph node ratio.

Age, IgFT3, IgTPO-Ab, tumor diameter $>1 \mathrm{~cm}$, multifocality, High $T$ stage and extrathyroidal extension as covariates to adjust OR value.

${ }^{1}$ The cut-off point of total LNR is 0.07 .

${ }^{2}$ The cut-off point of central LNR is 0.12 .

${ }^{3}$ The cut-off point of lateral LNR is 0.05 .

${ }^{*} P<0.05,{ }^{* *} P<0.01$.

After adjusting for confounding factors, it was found that overweight and obesity were independent risk factor for metastatic LNs $\left(\mathrm{OR}_{1}=1.125,95 \%\right.$ CI 1.042-1.214, $P_{1}=0.003$; $\mathrm{OR}_{2}=1.554,95 \%$ CI 1.339-1.802, $\left.P_{2}<0.001\right)$. This effect exists in both the central and lateral neck regions (Figure 3).

\section{Number of Metastatic LNs and the Sizes of Positive LNs}

The 2015 ATA guidelines suggest that more than five metastatic LNs yields an intermediate risk of recurrence (11). Previously, Leboulleux et al. found a recurrence rate of $3 \%$ with less than five metastases, while 6 to 10 metastases was associated with a recurrence rate of $7 \%$ and $>10$ metastases with $21 \%$ (31). Previous studies have given minimal attention to the relationship between the number of metastatic LNs and obesity. The current study revealed a positive correlation between obesity and metastatic LNs (2.41 vs. $1.79, P<0.001$ ), which was reflected in the central and lateral neck LNs (Figure 3).

The maximum diameter of positive LNs is another indicator of poor prognoses. The ATA guidelines classify the maximum diameter of positive $\mathrm{LN}$ between 0.2 and $3.0 \mathrm{~cm}$ as the intermediate risk of recurrence (11). In a previous study, the rate of locoregional recurrence was $5 \%$ for patients with maximum positive LN diameters smaller than $0.2 \mathrm{~cm}$ (12). When the maximum diameter of positive LNs were $>3 \mathrm{~cm}$, the rate of locoregional recurrence increased to $27 \%$ (13). In the current study, obese patients with PTC had larger maximum positive LN diameters (2.00 vs. $1.60, P=0.007)$, suggesting that obesity may lead to poorer prognoses. Previous studies have primarily shown that obesity increases the rate of LN metastasis. This paper confirmed that obesity not only increases the rate of metastatic LNs, but also increases the number of metastatic LNs and the maximum diameter of positive LNs.

\section{Number of Dissected LN}

Neck LNs are commonly wrapped in adipose tissue, and obese patients have more adipose tissue. Only a few studies have focused on whether the increased adipose tissue in obese patients can affect the dissection of neck LNs. This study found that obese patients had more dissectible LNs, yet this association only exists in the lateral neck area (14.37 vs. $12.10, P<0.001)$, which seems to be more beneficial for obese patients. However, the number of CNDs
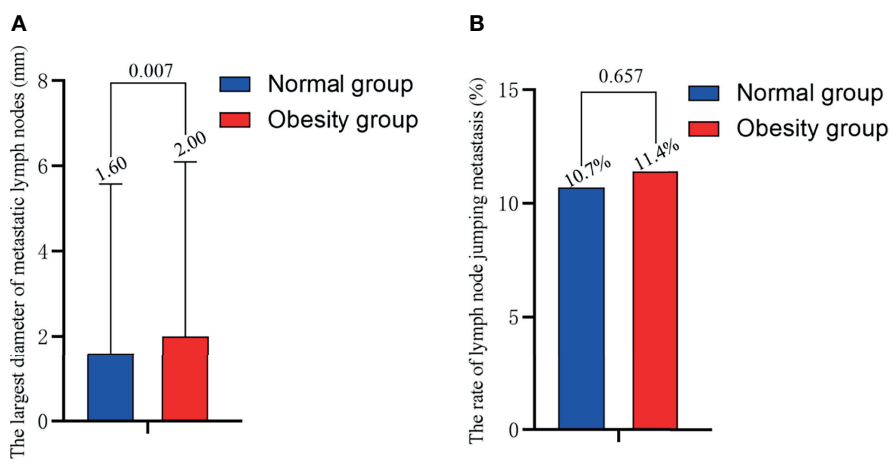

FIGURE 2 | Relationship of obesity with (A) the maximum diameter of positive LN and (B) the rate of LN skip metastasis. BMI, body mass index; LN, lymph node. 


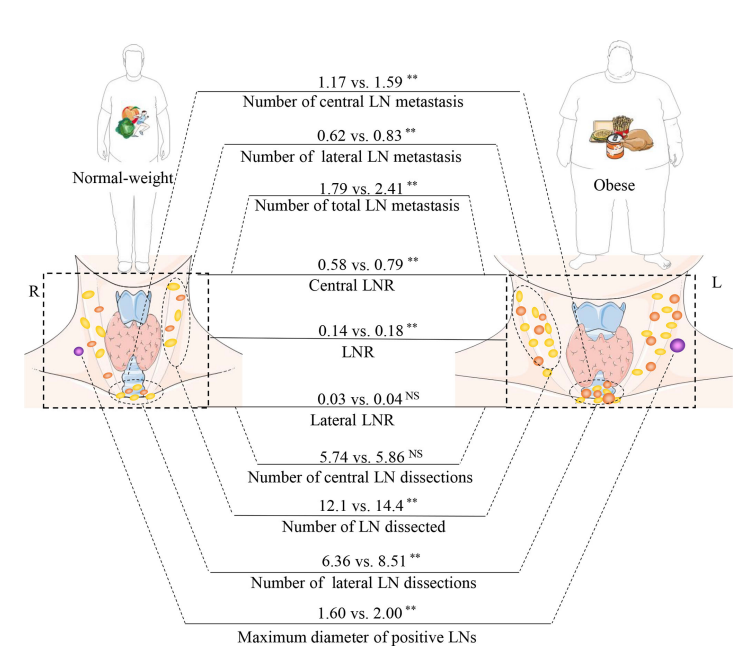

FIGURE 3 | Relationship between obesity and the rules of lymph node (LN) metastasis. The part of schematic art pieces used in this figure were provided by Servier Medical art (http://servier.com/Powerpoint-image-bank). Servier Medical Art by Servier is licensed under a Creative Commons Attribution (CC BY) 3.0 Unported License. BMI, body mass index; LNR, Lymph node ratio; $\mathrm{LN}$, lymph node. ${ }^{\star \star} \mathrm{P}<0.01$; ns, not statistically significant.

is not related to obesity. There is a regional difference between the number of dissected LN and obesity (Figure 3). One of the possible reasons is that the difficulty of intraoperative identification of LNs is reduced. LNs are often surrounded by adipose tissue, which helps the surgeon identify the LNs and minimize the burden of identification. The second reason involves inflammatory factor stimulation, as obesity can cause adipose cells to secrete inflammatory factors, such as C-reactive protein, interleukin 6 (IL-6), IL-10, and tumor necrosis factor (TNF- $\alpha$ ). These inflammatory factors may stimulate LN hyperplasia (32).

\section{LNR}

After discovering a positive relationship between obesity and the number of lateral neck dissected $L N$, we speculated whether more dissected LN would yield higher positivity rates, which could contribute to the radical cure of thyroid cancer in obese patients. However, by analyzing the LNR, we found that obesity did not increase the positive rate of lateral central LNs. On the other hand, obesity was found to increase the positive rate of central LNs. Hence, there are regional differences in the LNR and the number of dissected $L N$.

Obese patients have difficulty in performing CNDs due to their short and thick necks. In this study, obesity was not associated with an increase in the number of CNDs, but it was associated with an increased LNR. Hence, surgeons should be cautious when performing CNDs for obese patients, as there may be more positive LNs in obese patients. Obesity may increase risk of inadequate lymph node dissection during surgery.

In the current study, we revealed that obesity promotes the metastasis of LNs and increases the average diameter of positive LNs. These two indicators are indicative of a poor prognosis. In addition, we considered the number of dissected LN and the LNR into the current analysis. We found a regional difference between obesity and these two indicators.

\section{Limitations}

Most sources of error due to confounding and bias are more common in retrospective studies than in prospective studies. However, this retrospective study will be helpful to assess the feasibility of future prospective studies and to help in their design. This paper did not analyze the relationship between obesity and LNs in various regions of the lateral neck. The number of positive nodes is often affected by the variability in nodal staging techniques, which may yield different numbers of excised nodes. Finally, this study did not analyze the disease-free survival rate or locoregional recurrence rate of patients, for which LN metastasis is a very important independent risk factor in patients with thyroid cancer after curative resection.

This is a large-scale study of more than 10.000 PTC patients in which several of significance tests were performed. The testing procedure can be biased by latent confounding factors such as batch effects and unmeasured covariates that correlate with both primary variables of interest and the outcome. Despite the methodological advances in this paper, providing analysis that are able to capture complex and regional shape differences,

TABLE 8 | Previous studies on the relationship between obesity and LN metastasis of thyroid cancer.

\begin{tabular}{|c|c|c|c|c|c|c|}
\hline References & Date & Race & Cases (N) & BMI $\left(\mathrm{kg} / \mathrm{m}^{2}\right)$ & Rate of obesity (\%) & Correlation between BMI and LN metastasis \\
\hline Paes et al. (18) & 2010 & Mostly Caucasian (93\%) & 259 & 27.8 & $38.9 \%^{1}$ & Negative correlation \\
\hline Kim et al. (19) & 2013 & Asian & 2057 & 23.8 & $5 \%^{1}$ & Non-correlation \\
\hline Tresallet et al. (20) & 2014 & Caucasian & 1216 & $\mathrm{~N} / \mathrm{A}$ & $14.5 \%^{1}$ & Non-correlation \\
\hline Lee et al. (21) & 2015 & Asian & 1121 & 23.3 & $27 \%^{1}$ & Non-correlation \\
\hline Choi et al. (22) & 2015 & Asian & 612 & 23.1 & $2.1 \%^{1}$ & Non-correlation \\
\hline Kim et al. (23) & 2016 & Asian & 5081 & $\mathrm{~N} / \mathrm{A}$ & $5 \%^{1}$ & Correlation \\
\hline Yu et al. (24) & 2017 & Asian & 1622 & $\mathrm{~N} / \mathrm{A}$ & $24.3 \%^{2}$ & Correlation \\
\hline Wu et al. (25) & 2017 & Asian & 796 & 25 & $8 \%^{1}$ & Correlation \\
\hline Gasior et al. (26) & 2018 & Caucasian & 1181 & 28.1 & $33.7 \%^{1}$ & Non-correlation \\
\hline Feng et al. (27) & 2013 & Asian & 417 & 23.9 & $6 \%^{1}$ & Correlation \\
\hline Grani et al. (28) & 2018 & Caucasian & 432 & $\mathrm{~N} / \mathrm{A}$ & $19.8 \%^{1}$ & Non-correlation \\
\hline
\end{tabular}

${ }^{1}$ Defined the standard of obesity as $B M I \geq 27.5 \mathrm{~kg} / \mathrm{m}^{2}$.

${ }^{2}$ Defined the standard of obesity as $B M I \geq 30.0 \mathrm{~kg} / \mathrm{m}^{2}$.

N/A, Not available. 
the limits of the analysis methodology may remain. It is clear, for example, that a limit and bias of this work is BMI remodelling of PTC patients due to the practice (or not practice) of exercise, which it is perhaps impossible to analyze with the present data available. In addition, these data represent the work of multiple surgeons with varying degree of expertise.

\section{DATA AVAILABILITY STATEMENT}

The raw data supporting the conclusions of this article will be made available by the authors, without undue reservation. Requests to access the datasets should be directed to Hui Sun, s_h@jlu.edu.cn.

\section{ETHICS STATEMENT}

This study was approved by the Health Care Ethics Committee of the China-Japan Union Hospital of Jilin University (No. 2019040806).

\section{REFERENCES}

1. NCD Risk Factor Collaboration (NCD-RisC)Trends in Adult Body-Mass Index in 200 Countries From 1975 to 2014: A Pooled Analysis of 1698 Population-Based Measurement Studies With 19.2 Million Participants. Lancet (2016) 387(10026):1377-96. doi: 10.1016/s0140-6736(16)30054-x

2. Keum N, Greenwood DC, Lee DH, Kim R, Aune D, Ju W, et al. Adult Weight Gain and Adiposity-Related Cancers: A Dose-Response Meta-Analysis of Prospective Observational Studies. J Natl Cancer Inst (2015) 107(2):1-14. doi: 10.1093/jnci/djv088

3. Chen W, Zheng R, Baade PD, Zhang S, Zeng H, Bray F, et al. Cancer Statistics in China, 2015. CA Cancer J Clin (2016) 66(2):115-32. doi: 10.3322/ caac. 21338

4. Eckel RH, Kahn SE, Ferrannini E, Goldfine AB, Nathan DM, Schwartz MW, et al. Obesity and Type 2 Diabetes: What Can Be Unified and What Needs to be Individualized? J Clin Endocrinol Metab (2011) 96(6):1654-63. doi: 10.1210/jc.2011-0585

5. Wiltshire JJ, Drake TM, Uttley L, Balasubramanian SP. Systematic Review of Trends in the Incidence Rates of Thyroid Cancer. Thyroid (2016) 26 (11):1541-52. doi: 10.1089/thy.2016.0100

6. Deglise C, Bouchardy C, Burri M, Usel M, Neyroud-Caspar I, Vlastos G, et al. Impact of Obesity on Diagnosis and Treatment of Breast Cancer. Breast Cancer Res Treat (2010) 120(1):185-93. doi: 10.1007/s10549-009-0459-1

7. Fussey JM, Beaumont RN, Wood AR, Vaidya B, Smith J, Tyrrell J. Does Obesity Cause Thyroid Cancer? A Mendelian Randomization Study. J Clin Endocrinol Metab (2020) 105(7):e2398-407. doi: 10.1210/clinem/dgaa250

8. Li CL, Dionigi G, Zhao YS, Liang N, Sun H. Influence of Body Mass Index on the Clinicopathological Features of 13,995 Papillary Thyroid Tumors. J Endocrinol Invest (2020) 43(9):1283-99. doi: 10.1007/s40618-020-01216-6

9. Li C, Kuang J, Zhao Y, Sun H, Guan H. Effect of Type 2 Diabetes and Antihyperglycemic Drug Therapy on Signs of Tumor Invasion in Papillary Thyroid Cancer. Endocrine (2020) 69(1):92-99. doi: 10.1007/s12020-02002291-8

10. Li C, Zhou L, Dionigi G, Li F, Zhao Y, Sun H. The Association Between Tumor Tissue Calcification, Obesity, and Thyroid Cancer Invasiveness in A Cohort Study. Endocr Pract (2020) 26(8):830-39. doi: 10.4158/EP-2020-0057

11. Haugen BR, Alexander EK, Bible KC, Doherty GM, Mandel SJ, Nikiforov YE, et al. 2015 American Thyroid Association Management Guidelines for Adult Patients With Thyroid Nodules and Differentiated Thyroid Cancer: The American Thyroid Association Guidelines Task Force on Thyroid Nodules

\section{AUTHOR CONTRIBUTIONS}

Conception and design, HS and HG. Administrative support, HS. Collection and assembly of data, CL and NL. Data analysis and interpretation, NL. Manuscript writing, CL and GD. All authors contributed to the article and approved the submitted version

\section{FUNDING}

This study was supported by the National Nature Science Foundation of China NSFC, Grant No: 81972499.

\section{ACKNOWLEDGMENTS}

We thank all the doctors and nurses of Division of Thyroid Surgery, China-Japan Union Hospital of Jilin University who have contributed so generously to the database from 2008 until the present time.

and Differentiated Thyroid Cancer. Thyroid (2016) 26(1):1-133. doi: 10.1089/ thy. 2015.0020

12. Cranshaw IM, Carnaille B. Micrometastases in Thyroid Cancer. An Important Finding? Surg Oncol (2008) 17(3):253-8. doi: 10.1016/j.suronc. 2008.04.005

13. Sugitani I, Kasai N, Fujimoto Y, Yanagisawa A. A Novel Classification System for Patients With PTC: Addition of the New Variables of Large $(3 \mathrm{Cm}$ or Greater) Nodal Metastases and Reclassification During the Follow-Up Period. Surgery (2004) 135(2):139-48. doi: 10.1016/S0039-6060(03)00384-2

14. Chinese Medical Association. Guidelines for Diagnosis and Treatment of Thyroid Nodules and Differentiated Thyroid Cancer. Chin J Clin Oncol (2012) 39:1249-72. doi: 10.3969/j.issn.1000-8179.2012.17.003

15. World Health Organization. Obesity: Preventing and Managing the Global Epidemic. Report of a WHO Consultation. World Health Organ Tech Rep Ser (2000) 894:i-xii, 1-253.

16. Huvos AG, Hutter RV, Berg JW. Significance of Axillary Macrometastases and Micrometastases in Mammary Cancer. Ann Surg (1971) 173(1):44-6. doi: 10.1097/00000658-197101000-00006

17. Amit M, Tam S, Boonsripitayanon M, Cabanillas ME, Busaidy NL, Grubbs EG, et al. Association of Lymph Node Density With Survival of Patients With Papillary Thyroid Cancer. JAMA Otolaryngol Head Neck Surg (2018) 144 (2):108-14. doi: 10.1001/jamaoto.2017.2416

18. Paes JE, Hua K, Nagy R, Kloos RT, Jarjoura D, Ringel MD. The Relationship Between Body Mass Index and Thyroid Cancer Pathology Features and Outcomes: A Clinicopathological Cohort Study. J Clin Endocrinol Metab (2010) 95(9):4244-50. doi: 10.1210/jc.2010-0440

19. Kim HJ, Kim NK, Choi JH, Sohn SY, Kim SW, Jin SM, et al. Associations Between Body Mass Index and Clinico-Pathological Characteristics of Papillary Thyroid Cancer. Clin Endocrinol (Oxf) (2013) 78(1):134-40. doi: 10.1111/j.1365-2265.2012.04506.x

20. Tresallet C, Seman M, Tissier F, Buffet C, Lupinacci RM, Vuarnesson H, et al. The Incidence of Papillary Thyroid Carcinoma and Outcomes in Operative Patients According to Their Body Mass Indices. Surgery (2014) 156(5):114552. doi: 10.1016/j.surg.2014.04.020

21. Lee J, Lee CR, Ku CR, Kang SW, Jeong JJ, Shin DY, et al. Association Between Obesity and BRAFV600E Mutation Status in Patients With Papillary Thyroid Cancer. Ann Surg Oncol (2015) 22 Suppl 3:S683-90. doi: 10.1245/s10434-015$4765-\mathrm{z}$

22. Choi JS, Kim EK, Moon HJ, Kwak JY. Higher Body Mass Index May be a Predictor of Extrathyroidal Extension in Patients With Papillary Thyroid 
Microcarcinoma. Endocrine (2015) 48(1):264-71. doi: 10.1007/s12020-0140293-Z

23. Kim SK, Woo JW, Park I, Lee JH, Choe JH, Kim JH, et al. Influence of Body Mass Index and Body Surface Area on the Behavior of Papillary Thyroid Carcinoma. Thyroid (2016) 26(5):657-66. doi: 10.1089/thy.2015.0632

24. Yu ST, Chen W, Cai Q, Liang F, Xu D, Han P, et al. Pretreatment BMI Is Associated With Aggressive Clinicopathological Features of Papillary Thyroid Carcinoma: A Multicenter Study. Int J Endocrinol (2017) 2017:5841942. doi: $10.1155 / 2017 / 5841942$

25. Wu C, Wang L, Chen W, Zou S, Yang A. Associations Between Body Mass Index and Lymph Node Metastases of Patients With Papillary Thyroid Cancer: A Retrospective Study. Medicine (Baltimore) (2017) 96(9):e6202. doi: 10.1097/MD.0000000000006202

26. Gasior-Perczak D, Palyga I, Szymonek M, Kowalik A, Walczyk A, Kopczynski J, et al. The Impact of BMI on Clinical Progress, Response to Treatment, and Disease Course in Patients With Differentiated Thyroid Cancer. PLoS One (2018) 13(10):e0204668. doi: 10.1371/journal.pone.0204668

27. Feng C, Gao Y, Wang C, Yu X, Zhang W, Guan H, et al. Aberrant Overexpression of Pyruvate Kinase M2 Is Associated With Aggressive Tumor Features and the BRAF Mutation in Papillary Thyroid Cancer. J Clin Endocrinol Metab (2013) 98(9):E1524-33. doi: 10.1210/jc.2012-4258

28. Grani G, Lamartina L, Montesano T, Ronga G, Maggisano V, Falcone R, et al. Lack of Association Between Obesity and Aggressiveness of Differentiated Thyroid Cancer. J Endocrinol Invest (2018) 42(1):85-90. doi: 10.1007/s40618018-0889-x

29. Cheng SP, Yin PH, Hsu YC, Chang YC, Huang SY, Lee JJ, et al. Leptin Enhances Migration of Human Papillary Thyroid Cancer Cells Through the PI3K/AKT and MEK/ERK Signaling Pathways. Oncol Rep (2011) 26(5):126571. doi: $10.3892 /$ or. 2011.1388
30. Cheng SP, Yin PH, Chang YC, Lee CH, Huang SY, Chi CW. Differential Roles of Leptin in Regulating Cell Migration in Thyroid Cancer Cells. Oncol Rep (2010) 23(6):1721-7. doi: 10.3892/or_00000817

31. Leboulleux S, Rubino C, Baudin E, Caillou B, Hartl DM, Bidart J-M, et al. Prognostic Factors for Persistent or Recurrent Disease of Papillary Thyroid Carcinoma With Neck Lymph Node Metastases and/or Tumor Extension Beyond the Thyroid Capsule at Initial Diagnosis. J Clin Endocrinol Metab (2005) 90(10):5723-9. doi: 10.1210/jc.2005-0285

32. Dossus L, Franceschi S, Biessy C, Navionis AS, Travis RC, Weiderpass E, et al. Adipokines and Inflammation Markers and Risk of Differentiated Thyroid Carcinoma: The EPIC Study. Int J Cancer (2018) 142(7):1332-42. doi: $10.1002 / \mathrm{ijc} .31172$

Conflict of Interest: The authors declare that the research was conducted in the absence of any commercial or financial relationships that could be construed as a potential conflict of interest.

Publisher's Note: All claims expressed in this article are solely those of the authors and do not necessarily represent those of their affiliated organizations, or those of the publisher, the editors and the reviewers. Any product that may be evaluated in this article, or claim that may be made by its manufacturer, is not guaranteed or endorsed by the publisher.

Copyright $\odot 2021$ Li, Dionigi, Liang, Guan and Sun. This is an open-access article distributed under the terms of the Creative Commons Attribution License (CC BY). The use, distribution or reproduction in other forums is permitted, provided the original author(s) and the copyright owner(s) are credited and that the original publication in this journal is cited, in accordance with accepted academic practice. No use, distribution or reproduction is permitted which does not comply with these terms. 